\title{
Realizable Galois module classes for tetrahedral extensions
}

\author{
Nigel P. Byott and Bouchaïb Sodaïgui
}

\begin{abstract}
Let $k$ be a number field with ring of integers $\mathfrak{O}_{k}$, and let $\Gamma=A_{4}$ be the tetrahedral group. For each tame Galois extension $N / k$ with group isomorphic to $\Gamma$, the ring of integers $\mathfrak{O}_{N}$ of $N$ determines a class in the locally free class group $\mathrm{Cl}\left(\mathfrak{O}_{k}[\Gamma]\right)$. We show that the set of classes in $\mathrm{Cl}\left(\mathfrak{O}_{k}[\Gamma]\right)$ realized in this way is the kernel of the augmentation homomorphism from $\mathrm{Cl}\left(\mathfrak{O}_{k}[\Gamma]\right)$ to the ideal class group $\mathrm{Cl}\left(\mathfrak{O}_{k}\right)$. This refines a result of Godin and Sodaïgui (J. Number Theory 98 (2003), 320-328) on Galois module structure over a maximal order in $k[\Gamma]$. To the best of our knowledge, our result gives the first case where the set of realizable classes in $\mathrm{Cl}\left(\mathfrak{O}_{k}[\Gamma]\right)$ has been determined for a nonabelian group $\Gamma$ and an arbitrary number field $k$.
\end{abstract}

\section{Introduction}

Let $k$ be a number field and $\Gamma$ a finite group. For any Galois extension $N$ of $k$ with group isomorphic to $\Gamma$, the ring of integers $\mathfrak{O}_{N}$ of $N$ may be viewed as a module over the group ring $\mathfrak{O}_{k}[\Gamma]$. If $N / k$ is at most tamely ramified (we abbreviate this to 'tame') then $\mathfrak{O}_{N}$ is locally free over $\mathfrak{O}_{k}[\Gamma]$ and determines a class $\left(\mathfrak{O}_{N}\right)$ in the class group $\mathrm{Cl}\left(\mathfrak{O}_{k}[\Gamma]\right)$ of locally free $\mathfrak{O}_{k}[\Gamma]$-modules. The subset $\mathcal{R}\left(\mathfrak{O}_{k}[\Gamma]\right)$ of realizable classes in $\mathfrak{O}_{k}[\Gamma]$ is defined as the set of all classes $\left(\mathfrak{O}_{N}\right)$ as $N$ runs through the tame Galois extensions of $k$ with group isomorphic to $\Gamma$. We expect that $\mathcal{R}\left(\mathfrak{O}_{k}[\Gamma]\right)$ is always a subgroup of $\mathrm{Cl}\left(\mathfrak{O}_{k}[\Gamma]\right)$, but this is far from obvious. Indeed a general proof that $\mathcal{R}\left(\mathfrak{O}_{k}[\Gamma]\right)$ is even nonempty would solve the inverse Galois problem for $k$.

In this paper, we take $\Gamma$ to be the tetrahedral group $A_{4}$ (i.e. the alternating group of degree 4 ). We determine the set $\mathcal{R}\left(\mathfrak{O}_{k}[\Gamma]\right)$ of realizable classes in this case, showing that it is indeed a subgroup of $\mathrm{Cl}\left(\mathfrak{O}_{k}[\Gamma]\right)$. To the best of our knowledge, this is the first case where $\mathcal{R}\left(\mathfrak{D}_{k}[\Gamma]\right)$ has been completely determined for a nonabelian group $\Gamma$ and for an arbitrary number field $k$. Elsewhere we give a similar result for the dihedral group of order 8 [BS05], but only under a technical restriction on $k$. (To be precise, the ray class group of $\mathfrak{O}_{k}$ with modulus $4 \mathfrak{O}_{k}$ was required to have odd order.)

We briefly review the background to this work. The investigation of realizable classes was initiated by McCulloh. In [McC83] he gave a concrete description of $\mathcal{R}\left(\mathfrak{O}_{k}[\Gamma]\right)$ for elementary abelian groups $\Gamma$, and subsequently he determined $\mathcal{R}\left(\mathfrak{O}_{k}[\Gamma]\right)$ in a less explicit form for arbitrary abelian groups [McC87]. Thus in the abelian case, $\mathcal{R}\left(\mathfrak{O}_{k}[\Gamma]\right)$ is known, at least in principle, and in fact $\mathcal{R}\left(\mathfrak{O}_{k}[\Gamma]\right)$ is always a subgroup of $\mathrm{Cl}\left(\mathfrak{O}_{k}[\Gamma]\right)$. For nonabelian groups, however, there are as yet only partial results in a few special cases. Some progress can be made by extending scalars from $\mathfrak{O}_{k}[\Gamma]$ to a maximal order $\mathfrak{M}$ in $k[\Gamma]$ containing $\mathfrak{O}_{k}[\Gamma]$. The resulting subset $\mathcal{R}(\mathfrak{M})$ of realizable classes in the class group $\mathrm{Cl}(\mathfrak{M})$ is easier to determine than $\mathcal{R}\left(\mathfrak{O}_{k}[\Gamma]\right)$, since the class in $\mathrm{Cl}(\mathfrak{M})$

Received 18 November 2003, accepted in final form 15 May 2004, published online 21 April 2005.

2000 Mathematics Subject Classification 11R33.

Keywords: Galois module structure, realizable classes, locally free class group.

This journal is (c) Foundation Compositio Mathematica 2005. 


\section{N. P. Byott And B. Sodä̈Gui}

of $\mathfrak{M} \otimes_{\mathfrak{O}_{k}[\Gamma]} \mathfrak{O}_{N}$ can be calculated from the Steinitz classes of the rings of integers in the various intermediate fields of $N / k$. This approach has been developed by the second-named author in a series of papers [Sod97, Sod99, Sod00, GS03]. The case of metacyclic groups has also been investigated in unpublished work of Soverchia [Sov99], but her results again fall short of a complete determination of the realizable classes over the group ring $\mathfrak{O}_{k}[\Gamma]$ itself.

Our treatment here of $\mathcal{R}\left(\mathfrak{O}_{k}[\Gamma]\right)$ for $\Gamma=A_{4}$ refines the analysis of $\mathcal{R}(\mathfrak{M})$ for $\Gamma=A_{4}$ given by Godin and Sodaïgui in [GS03]. Thus the present paper bears the same relationship to [GS03] as [BS05] does to [Sod00]. In [GS03] it was assumed that $k$ has odd class number and does not contain a primitive cube root of unity. These hypotheses are not required in the present paper.

To state our main result, we introduce some more notation. The augmentation map $\varepsilon$ : $\mathfrak{O}_{k}[\Gamma] \longrightarrow \mathfrak{O}_{k}$ induces a group homomorphism $\mathrm{Cl}\left(\mathfrak{O}_{k}[\Gamma]\right) \longrightarrow \mathrm{Cl}\left(\mathfrak{O}_{k}\right)$, where $\mathrm{Cl}\left(\mathfrak{O}_{k}\right)$ is the ideal class group of $\mathfrak{O}_{k}$. We write $\mathrm{Cl}^{0}\left(\mathfrak{O}_{k}[\Gamma]\right)$ for the kernel of this homomorphism. It is easily seen that

$$
\mathcal{R}\left(\mathfrak{O}_{k}[\Gamma]\right) \subseteq \mathrm{Cl}^{0}\left(\mathfrak{O}_{k}[\Gamma]\right)
$$

(cf. $[\mathrm{McC} 83,(4.4)]$ ): this is simply a restatement of the fact that $\operatorname{Tr}_{N / k}\left(\mathfrak{O}_{N}\right)$ is a principal $\mathfrak{O}_{k}$-ideal (actually $\mathfrak{O}_{k}$ itself) for any tame extension $N / k$. Here $\operatorname{Tr}_{N / k}$ denotes the trace map. Our main result is that the inclusion (1.1) is an equality, expressed in the following theorem.

TheOREm. Let $k$ be any number field and let $\Gamma=A_{4}$. Then $\mathcal{R}\left(\mathfrak{O}_{k}[\Gamma]\right)$ is the subgroup $\operatorname{Cl}^{0}\left(\mathfrak{O}_{k}[\Gamma]\right)$ of $\mathrm{Cl}\left(\mathfrak{O}_{k}[\Gamma]\right)$.

At the suggestion of one of the referees, we briefly mention some unpublished work of McCulloh on realizable classes for nonabelian groups. One formulation of McCulloh's main result in the abelian case is that the set of realizable classes is precisely the kernel of a certain homomorphism $\operatorname{Rag}^{\prime}$ on $\mathrm{Cl}\left(\mathfrak{O}_{k}[\Gamma]\right)$ (see [McC87, p. 291]). Using the Hom-description of the class group, the definition of $\operatorname{Rag}^{\prime}$ carries over to the nonabelian case once one extends the Stickelberger pairing [McC87, (4.1)(b)] from abelian characters to arbitrary characters by setting $\langle\chi, s\rangle=\left\langle\operatorname{res}_{\langle s\rangle}^{G} \chi, s\right\rangle$ for any irreducible character $\chi$ of $G$ and any $s \in G$. McCulloh has shown that for an arbitrary finite group $\Gamma$ we have $\mathcal{R}\left(\mathfrak{O}_{k}[\Gamma]\right) \subseteq \operatorname{ker}\left(R a g^{\prime}\right)$. One can verify that $\operatorname{ker}\left(\operatorname{Rag}^{\prime}\right)=\mathrm{Cl}^{0}\left(\mathfrak{O}_{k}[\Gamma]\right)$ in the case $\Gamma=A_{4}$. The main point in the present paper is to prove the opposite inclusion $\mathcal{R}\left(\mathfrak{O}_{k}[\Gamma]\right) \supseteq \operatorname{Cl}^{0}\left(\mathfrak{O}_{k}[\Gamma]\right)$ in this case.

\section{The Hom-description}

For the rest of this paper, we fix $\Gamma=A_{4}$, the tetrahedral group of order 12 . We work with the presentation

$$
\Gamma=\left\langle\sigma, \tau, \nu \mid \sigma^{3}=\tau^{2}=\nu^{2}=1, \tau \nu=\nu \tau, \sigma \tau \sigma^{-1}=\nu, \sigma \nu \sigma^{-1}=\tau \nu\right\rangle .
$$

Let $\Delta$ denote the normal subgroup $\langle\tau, \nu\rangle$ of $\Gamma$ isomorphic to $C_{2} \times C_{2}$.

The absolutely irreducible characters of $\Gamma$ are the trivial character $\chi_{0}$, two nontrivial characters $\chi_{1}$ and $\chi_{1}^{2}$ of degree 1 , and a character $\chi_{2}=\operatorname{Ind}_{\Delta}^{\Gamma} \phi$ of degree 3 induced from any nontrivial irreducible character $\phi$ of $\Delta$. The character $\chi_{1}$ factors through $\Gamma / \Delta$, and is determined by $\chi_{1}(\sigma)=\zeta$ for a fixed primitive cube root $\zeta$ of unity. Without loss of generality, we take $\phi$ to have kernel $\langle\nu\rangle$. The characters $\chi_{0}$ and $\chi_{2}$ are always defined over $k$, whereas $\chi_{1}$ is defined over $k$ only if $\zeta \in k$. To treat the two cases $\zeta \in k$ and $\zeta \notin k$ in a uniform manner, we set

$$
K= \begin{cases}k \times k & \text { if } \zeta \in k ; \\ k(\zeta) & \text { if } \zeta \notin k .\end{cases}
$$

Then $k[\Gamma]$ has Wedderburn decomposition $k \times K \times M_{3}(k)$. 


\section{Realizable Galois module Classes}

We recall Fröhlich's so-called Hom-description of $\mathrm{Cl}\left(\mathfrak{D}_{k}[\Gamma]\right)$ in terms of Galois-equivariant functions on the group $R_{\Gamma}$ of virtual characters of $\Gamma$. From [Frö83, p. 20] we have

$$
\mathrm{Cl}\left(\mathfrak{O}_{k}[\Gamma]\right) \cong \frac{\operatorname{Hom}_{\Omega}\left(R_{\Gamma}, \mathbb{J}\left(k^{c}\right)\right)}{\operatorname{Hom}_{\Omega}\left(R_{\Gamma}, k^{c \times}\right) \operatorname{Det}\left(\mathbb{U}\left(\mathfrak{O}_{k}[\Gamma]\right)\right)},
$$

where $k^{c}$ is an algebraic closure of $k, \Omega=\operatorname{Gal}\left(k^{c} / k\right)$ the absolute Galois group of $k, \mathbb{J}\left(k^{c}\right)$ the idèle group of $k^{c}$ (that is, the limit of the idèle groups $\mathbb{J}(L)$ as $L$ runs over all finite extensions of $k$ inside $\left.k^{c}\right), \mathbb{U}\left(\mathfrak{O}_{k}[\Gamma]\right)$ the group of unit idèles of $\mathfrak{O}_{k}[\Gamma]$, and Det : $\mathbb{U}\left(\mathfrak{O}_{k}[\Gamma]\right) \longrightarrow \operatorname{Hom}_{\Omega}\left(R_{\Gamma}, \mathbb{J}\left(k^{c}\right)\right)$ the determinant map given by $\operatorname{Det}(\alpha)(\chi)=\operatorname{det}\left(T_{\chi}(\alpha)\right)$ for any representation $T_{\chi}$ affording the character $\chi$. For a tame Galois extension $N / k$ with group $\Gamma$, the class $\left(\mathfrak{O}_{N}\right) \in \operatorname{Cl}\left(\mathfrak{O}_{k}[\Gamma]\right)$ is obtained in terms of the Hom-description as follows (see [Frö83, p. 30]). Let $\eta \in N$ be a normal basis for $N / k$, so $N=k[\Gamma] \eta$. For an irreducible character $\chi$ of $\Gamma$, form the Fröhlich-Lagrange resolvent

$$
\langle\eta, \chi\rangle_{N / k}=\operatorname{det}\left(\sum_{\gamma \in \Gamma} \gamma(\eta) T_{\chi}\left(\gamma^{-1}\right)\right) .
$$

For each place $\mathfrak{p}$ of $\mathfrak{O}_{k}$, the semilocal completion $\mathfrak{O}_{N, \mathfrak{p}}$ of $\mathfrak{O}_{N}$ at $\mathfrak{p}$ is a free $\mathfrak{O}_{k, \mathfrak{p}}[\Gamma]$-module since $N / k$ is tame. Let $\eta_{\mathfrak{p}} \in \mathfrak{O}_{N, \mathfrak{p}}$ be such that $\mathfrak{O}_{N, \mathfrak{p}}=\mathfrak{O}_{k, \mathfrak{p}}[\Gamma] \eta_{\mathfrak{p}}$. We will refer to such an element as a local normal integral basis for $N / k$. Forming the local resolvents $\left\langle\eta_{\mathfrak{p}}, \chi\right\rangle_{N / k}$ for all $\mathfrak{p}$, we obtain an idèlic resolvent

$$
\left(\left\langle\eta_{\mathfrak{p}}, \chi\right\rangle_{N / k}\right)_{\mathfrak{p}} \in \mathbb{J}\left(k^{c}\right) .
$$

The assignment $\chi \mapsto\left(\left\langle\eta_{\mathfrak{p}}, \chi\right\rangle_{N / k}\right)_{\mathfrak{p}}\langle\eta, \chi\rangle_{N / k}^{-1}$ extends by linearity to an element of $\operatorname{Hom}_{\Omega}\left(R_{\Gamma}, \mathbb{J}\left(k^{c}\right)\right)$. The class of this element on the right in (2.1) is independent of the choices of $\eta$ and the $\eta_{\mathfrak{p}}$, and coincides with $\left(\mathfrak{O}_{N}\right)$.

As explained in [BS05], there is a similar Hom-description of the augmentation kernel:

$$
\mathrm{Cl}^{0}\left(\mathfrak{O}_{k}[\Gamma]\right) \cong \frac{\operatorname{Hom}_{\Omega}^{0}\left(R_{\Gamma}, \mathbb{J}\left(k^{c}\right)\right)}{\operatorname{Hom}_{\Omega}^{0}\left(R_{\Gamma}, k^{c \times}\right) \operatorname{Det}^{0}\left(\mathbb{U}\left(\mathfrak{O}_{k}[\Gamma]\right)\right)},
$$

where, for each of the three groups on the right, the superscript 0 indicates that only the homomorphisms $f$ with $f\left(\chi_{0}\right)=1$ are included. The class $\left(\mathfrak{O}_{N}\right)$ in $\operatorname{Cl}^{0}\left(\mathfrak{O}_{k}[\Gamma]\right)$ is obtained as described above, but with the normal basis $\eta$ and local normal integral bases $\eta_{\mathfrak{p}}$ chosen to satisfy $\operatorname{Tr}_{N / k}(\eta)=1$ and $\operatorname{Tr}_{N_{\mathfrak{p}} / k_{\mathfrak{p}}}\left(\eta_{\mathfrak{p}}\right)=1$ for all $\mathfrak{p}$.

In our case $\Gamma=A_{4}$, either the irreducible characters are all fixed by $\Omega$ (if $\zeta \in k$ ), or $\chi_{1}$ and $\chi_{1}^{2}$ are interchanged by $\Omega$ (if $\zeta \notin k$ ). In the first case we may identify any $f \in \operatorname{Hom}_{\Omega}^{0}\left(R_{\Gamma}, k^{c}\right.$ ) with its triple of values on nontrivial characters,

$$
\left(f\left(\chi_{1}\right), f\left(\chi_{1}^{2}\right), f\left(\chi_{2}\right)\right) \in\left(k^{\times}\right)^{3} .
$$

In the second case, $f\left(\chi_{1}\right) \in k(\zeta)^{\times}$and $f\left(\chi_{1}^{2}\right)=f\left(\chi_{1}\right)^{\omega}$ for any $\omega \in \Omega$ with $\zeta^{\omega}=\zeta^{2}$, so we may identify $f$ with the pair of values

$$
\left(f\left(\chi_{1}\right), f\left(\chi_{2}\right)\right) \in k(\zeta)^{\times} \times k^{\times} .
$$

Thus

$$
\operatorname{Hom}_{\Omega}^{0}\left(R_{\Gamma}, k^{c \times}\right)=K^{\times} \times k^{\times}
$$

in both cases. Similarly, we identify $\operatorname{Hom}_{\Omega}^{0}\left(R_{\Gamma}, \mathbb{J}\left(k^{c}\right)\right)$ with $\mathbb{J}(K) \times \mathbb{J}(k)$, and $\operatorname{Det}^{0}\left(\mathbb{U}\left(\mathfrak{O}_{k}[\Gamma]\right)\right)$ with a subgroup of $\mathbb{J}(K) \times \mathbb{J}(k)$. The Hom-description (2.2) then becomes

$$
\mathrm{Cl}^{0}\left(\mathfrak{O}_{k}[\Gamma]\right) \cong \frac{\mathbb{J}(K) \times \mathbb{J}(k)}{\left(K^{\times} \times k^{\times}\right) \operatorname{Det}^{0}\left(\mathbb{U}\left(\mathfrak{O}_{k}[\Gamma]\right)\right)} .
$$




\section{N. P. Byott AND B. SodaÏGui}

For a nonzero ideal $\mathfrak{f}$ of $\mathfrak{O}_{k}$, we define the ray class group $\mathrm{Cl}_{\mathfrak{f}}\left(\mathfrak{O}_{k}\right)$ by

$$
\mathrm{Cl}_{\mathfrak{f}}\left(\mathfrak{O}_{k}\right)=\frac{\mathbb{J}(k)}{k^{\times} \mathbb{U}_{f}\left(\mathfrak{O}_{k}\right)}
$$

where

$$
\mathbb{U}_{\mathfrak{f}}\left(\mathfrak{O}_{k}\right)=\left\{u \in \mathbb{U}\left(\mathfrak{O}_{k}\right) \mid u \equiv 1\left(\bmod ^{*} \mathfrak{f}\right)\right\} .
$$

We extend this notation to ray class groups of the integral closure $\mathfrak{O}_{K}$ of $\mathfrak{O}_{k}$ in $K$. An invertible $\mathfrak{O}_{K}$-ideal $\mathfrak{f}$ is either a nonzero ideal in the integral domain $\mathfrak{O}_{K}$ (if $\zeta \notin k$ ) or of the form $\mathfrak{f}_{1} \times \mathfrak{f}_{2}$ for nonzero ideals $\mathfrak{f}_{1}, \mathfrak{f}_{2}$ of $\mathfrak{O}_{k}$ (if $\zeta \in k$ ). We define $\mathrm{Cl}_{\mathfrak{f}}\left(\mathfrak{O}_{K}\right)$ to be the ray class group in the above sense in the first case, and set

$$
\mathrm{Cl}_{\mathfrak{f}}\left(\mathfrak{O}_{K}\right)=\mathrm{Cl}_{\mathfrak{f}_{1}}\left(\mathfrak{O}_{k}\right) \times \mathrm{Cl}_{\mathfrak{f}_{2}}\left(\mathfrak{O}_{k}\right)
$$

in the second.

Proposition 2.1. The natural map $\mathbb{J}(K) \times \mathbb{J}(k) \rightarrow \mathrm{Cl}^{0}\left(\mathfrak{D}_{k}[\Gamma]\right)$ given by (2.3) induces a surjection $\mathrm{Cl}_{\mathfrak{f}}\left(\mathfrak{O}_{K}\right) \times \mathrm{Cl}_{8}\left(\mathfrak{O}_{k}\right) \rightarrow \mathrm{Cl}^{0}\left(\mathfrak{O}_{k}[\Gamma]\right)$, for some ideal $\mathfrak{f}$ of $\mathfrak{O}_{K}$ divisible only by primes of $\mathfrak{O}_{k}$ above 2 and 3 .

Proof. For all finite places $\mathfrak{p}$ of $\mathfrak{O}_{k}$ not above 2 or 3 , we have $\operatorname{Det}^{0}\left(\mathfrak{O}_{k, \mathfrak{p}}[\Gamma]^{\times}\right)=\mathfrak{O}_{K, \mathfrak{p}}^{\times} \times \mathfrak{O}_{k, \mathfrak{p}}^{\times}$since $\mathfrak{O}_{k, \mathfrak{p}}[\Gamma]$ is a maximal order in $k_{\mathfrak{p}}[G]$. For the infinite places $\mathfrak{p}$ we have $\operatorname{Det}\left(k_{\mathfrak{p}}[\Gamma]^{\times}\right)=K_{\mathfrak{p}}^{\times} \times k_{\mathfrak{p}}^{\times}$ since none of the irreducible characters of $\Gamma$ are symplectic. It follows that there are ideals $\mathfrak{f}, \mathfrak{g}$ of $\mathfrak{O}_{K}, \mathfrak{O}_{k}$, respectively, divisible only by primes above 2 and 3 , such that

$$
\mathbb{U}_{\mathfrak{f}}\left(\mathfrak{O}_{K}\right) \times \mathbb{U}_{\mathfrak{g}}\left(\mathfrak{O}_{k}\right) \subseteq \operatorname{Det}^{0}\left(\mathbb{U}\left(\mathfrak{O}_{k}[\Gamma]\right)\right) .
$$

The Hom-description then induces a surjection $\mathrm{Cl}_{\mathfrak{f}}\left(\mathfrak{O}_{K}\right) \times \mathrm{Cl}_{\mathfrak{g}}\left(\mathfrak{O}_{k}\right) \rightarrow \mathrm{Cl}^{0}\left(\mathfrak{O}_{k}[\Gamma]\right)$. It remains to show that we may take $\mathfrak{g}=8 \mathfrak{O}_{k}$. Thus we have to verify that

$$
\left(1,1+8 \mathfrak{O}_{k, \mathfrak{p}}\right) \subseteq \operatorname{Det}^{0}\left(\mathfrak{O}_{k, \mathfrak{p}}[\Gamma]^{\times}\right) \text {for each place } \mathfrak{p} \text { above } 2,
$$

and that

$$
\left(1, \mathfrak{O}_{k, \mathfrak{p}}^{\times}\right) \subseteq \operatorname{Det}^{0}\left(\mathfrak{O}_{k, \mathfrak{p}}[\Gamma]^{\times}\right) \text {for each place } \mathfrak{p} \text { above } 3 .
$$

Now the character $\chi_{2}$ is afforded by the representation

$$
\sigma \mapsto\left(\begin{array}{lll}
0 & 0 & 1 \\
1 & 0 & 0 \\
0 & 1 & 0
\end{array}\right), \quad \tau \mapsto\left(\begin{array}{rrr}
-1 & 0 & 0 \\
0 & -1 & 0 \\
0 & 0 & 1
\end{array}\right), \quad \nu \mapsto\left(\begin{array}{rrr}
1 & 0 & 0 \\
0 & -1 & 0 \\
0 & 0 & -1
\end{array}\right) .
$$

For any place $\mathfrak{p}$ and any $a \in \mathfrak{O}_{k, \mathfrak{p}}$ such that $1+a(\tau-1) \in \mathfrak{O}_{k, \mathfrak{p}}[\Gamma]^{\times}$, we therefore have

$$
\left(1,(1-2 a)^{2}\right)=\operatorname{Det}(1+a(\tau-1)) \in \operatorname{Det}^{0}\left(\mathfrak{O}_{k, \mathfrak{p}}[\Gamma]^{\times}\right) .
$$

For $\mathfrak{p}$ above 2 , we may take any $a \in \mathfrak{O}_{k, \mathfrak{p}}$. Thus $\left(1,\left(1+2 \mathfrak{O}_{k, \mathfrak{p}}\right)^{2}\right) \subset \operatorname{Det}^{0}\left(\mathfrak{O}_{k, \mathfrak{p}}[\Gamma]^{\times}\right)$by $(2.6)$, and (2.4) follows since $1+8 \mathfrak{O}_{k, \mathfrak{p}} \subseteq\left(1+2 \mathfrak{O}_{k, \mathfrak{p}}\right)^{2}$.

For $\mathfrak{p}$ above 3 , we first observe that

$$
\left(1+\mathfrak{p} \mathfrak{O}_{k, \mathfrak{p}}\right)\left(\mathfrak{O}_{k, \mathfrak{p}}^{\times}\right)^{3}=\mathfrak{O}_{k, \mathfrak{p}}^{\times},
$$

since the function $x \mapsto x^{3}$ is surjective on the units of the residue field $\mathfrak{O}_{k} / \mathfrak{p}$. Now $\alpha=1+a(\tau-1)$ lies in $\mathfrak{O}_{k, \mathfrak{p}}[\Gamma]^{\times}$for any $a \in \mathfrak{p} \mathfrak{O}_{k, \mathfrak{p}}$. Thus, since

$$
\left\{(1-2 a)^{2} \mid a \in \mathfrak{p} \mathfrak{O}_{k, \mathfrak{p}}\right\}=1+\mathfrak{p} \mathfrak{O}_{k, \mathfrak{p}},
$$

it follows from $(2.6)$ that $\left(1,1+\mathfrak{p} \mathfrak{O}_{k, \mathfrak{p}}\right) \subseteq \operatorname{Det}^{0}\left(\mathfrak{O}_{k, \mathfrak{p}}[\Gamma]^{\times}\right)$. On the other hand, the idempotent

$$
e_{0}=\frac{1}{4}(1+\tau+\nu+\tau \nu) \in k[\Gamma]
$$


lies in $\mathfrak{O}_{k, \mathfrak{p}}[\Gamma]$, and for each $b \in \mathfrak{O}_{k, \mathfrak{p}}^{\times}$we have $\beta=e_{0}+b\left(1-e_{0}\right) \in \mathfrak{O}_{k, \mathfrak{p}}[\Gamma]^{\times}$. Thus

$$
\left(1, b^{3}\right)=\operatorname{Det}(\beta) \in \operatorname{Det}^{0}\left(\mathfrak{O}_{k, \mathfrak{p}}[\Gamma]^{\times}\right) .
$$

Now (2.5) follows from (2.7).

\section{Construction of the tetrahedral extension $N / k$}

To prove the Theorem, it suffices by (1.1) to show that any given class in $\mathrm{Cl}^{0}\left(\mathfrak{O}_{k}[\Gamma]\right)$ can be obtained as the class $\left(\mathfrak{O}_{N}\right)$ for some tame Galois extension $N / k$ with group $\Gamma=A_{4}$. Any class in $\mathrm{Cl}^{0}\left(\mathfrak{O}_{k}[\Gamma]\right)$ is represented under the Hom-description $(2.3)$ by a pair of idèles $(x, y) \in \mathbb{J}(K) \times \mathbb{J}(k)$. By Proposition 2.1, we may multiply $x$ by an element of $K^{\times} \mathbb{U}_{\mathfrak{f}}\left(\mathfrak{O}_{K}\right)$, and $y$ by an element of $k^{\times} \mathbb{U}_{8}\left(\mathfrak{O}_{k}\right)$, without changing the corresponding class in $\mathrm{Cl}^{0}\left(\mathfrak{O}_{k}[\Gamma]\right)$.

In this section we start with such a pair $(x, y)$ and construct a tame extension $N / k$. In the subsequent two sections, we will show that the class $\left(\mathfrak{O}_{N}\right)$ in $\mathrm{Cl}^{0}\left(\mathfrak{O}_{k}[\Gamma]\right)$ is indeed the class represented by $(x, y)$.

First consider the quotient group $C_{3}=A_{4} / \Delta$, generated by the image $\bar{\sigma}$ of $\sigma$. For each character $\chi$ of $A_{4}$ which factors through $C_{3}$, we write $\chi$ for the corresponding character of $C_{3}$. As in (2.3), we have a Hom-description

$$
\mathrm{Cl}^{0}\left(\mathfrak{O}_{k}\left[C_{3}\right]\right) \cong \frac{\mathbb{J}(K)}{K^{\times} \operatorname{Det}^{0}\left(\mathbb{U}\left(\mathfrak{O}_{k}\left[C_{3}\right]\right)\right)}
$$

By McCulloh's result [McC83], together with the fact that the Stickelberger ideal in $\mathbb{Z}[C]$, where $C=\operatorname{Aut}\left(C_{3}\right)=C_{2}$, is in fact the whole of $\mathbb{Z}[C]$ (see e.g. [GS03, p. 326]), we have $\mathcal{R}\left(\mathfrak{D}_{k}\left[C_{3}\right]\right)=$ $\mathrm{Cl}^{0}\left(\mathfrak{O}_{k}\left[C_{3}\right]\right)$. Indeed, by [McC83, Theorem 5.1], there exists a tame cubic Galois extension $E$ of $k$, ramified in at least one place, for which the class $\left(\mathfrak{O}_{E}\right) \in \mathrm{Cl}^{0}\left(\mathfrak{O}_{k}\left[C_{3}\right]\right)$ is represented by $x \in \mathbb{J}(K)$.

Since $E / k$ is tame, we know that $\mathfrak{O}_{E}$ is a locally free $\mathfrak{O}_{k}\left[C_{3}\right]$-module and $\operatorname{Tr}_{E / k}\left(\mathfrak{O}_{E}\right)=\mathfrak{O}_{k}$. For each place $\mathfrak{p}$ we choose a local normal integral basis $c_{\mathfrak{p}}^{\prime} \in \mathfrak{O}_{E, \mathfrak{p}}$. For $\mathfrak{p}$ not above 2, we replace $c_{\mathfrak{p}}^{\prime}$ by $c_{\mathfrak{p}}=c_{\mathfrak{p}}^{\prime}\left(\operatorname{Tr}_{E / k}\left(c_{\mathfrak{p}}^{\prime}\right)\right)^{-1}$, so that

$$
\mathfrak{O}_{E, \mathfrak{p}}=\mathfrak{O}_{k, \mathfrak{p}}\left[C_{3}\right] c_{\mathfrak{p}} \text { and } \operatorname{Tr}_{E_{\mathfrak{p}} / k_{\mathfrak{p}}}\left(c_{\mathfrak{p}}\right)=1 .
$$

We next choose $c^{\prime} \in E$ sufficiently close to $c_{\mathfrak{p}}^{\prime}$, for each of the finitely many places $\mathfrak{p}$ above 2 , to ensure that $c^{\prime}$ is a local normal integral basis at these places. Set $c=c^{\prime}\left(\operatorname{Tr}_{E / k}\left(c^{\prime}\right)\right)^{-1}$ and $c_{\mathfrak{p}}=c^{\prime}$ for all $\mathfrak{p}$ above 2. Then

and (3.2) now holds for all $\mathfrak{p}$.

$$
E=k\left[C_{3}\right] c \text { and } \operatorname{Tr}_{E / k}(c)=1
$$

The class $\left(\mathfrak{O}_{E}\right)$ is represented in the Hom-description (3.1) for $\mathrm{Cl}^{0}\left(\mathfrak{O}_{k}\left[C_{3}\right]\right)$ by the idèle $x^{\prime} \in \mathbb{J}(K)$ defined by

$$
x^{\prime}= \begin{cases}\left(\left(\left\langle c_{\mathfrak{p}}, \underline{\chi}_{1}\right\rangle_{E / k}\right)_{\mathfrak{p}}\left\langle c, \underline{\chi}_{1}\right\rangle_{E / k}^{-1},\left(\left\langle c_{\mathfrak{p}}, \underline{\chi}_{1}^{2}\right\rangle_{E / k}\right)_{\mathfrak{p}}\left\langle c, \underline{\chi}_{1}^{2}\right\rangle_{E / k}^{-1}\right) & \text { if } \zeta \in k, \\ \left(\left\langle c_{\mathfrak{p}}, \underline{\chi}_{1}\right\rangle_{E / k}\right)_{\mathfrak{p}}\left\langle c, \underline{\chi}_{1}\right\rangle_{E / k}^{-1} & \text { if } \zeta \notin k .\end{cases}
$$

Proposition 3.1. With the above notation, there exists $y^{\prime} \in \mathbb{J}(k)$ such that $\left(x^{\prime}, y^{\prime}\right)$ represents the same class in $\mathrm{Cl}^{0}\left(\mathfrak{O}_{k}[\Gamma]\right)$ as $(x, y)$.

Proof. By the choice of $E,\left(\mathfrak{O}_{E}\right)$ is also represented by $x$. Thus from (3.1) we have

$$
x^{-1} x^{\prime}=\alpha\left(\operatorname{Det}\left(\kappa_{\mathfrak{p}}\right)\right)_{\mathfrak{p}}
$$

for some $\alpha \in K^{\times}$and some elements $\kappa_{\mathfrak{p}} \in \mathfrak{O}_{k, \mathfrak{p}}\left[C_{3}\right]^{\times}$with $\operatorname{Det}\left(\kappa_{\mathfrak{p}}\right)\left(\chi_{0}\right)=1$. Lift each

$$
\kappa_{\mathfrak{p}}=a_{0, \mathfrak{p}}+a_{1, \mathfrak{p}} \bar{\sigma}+a_{2, \mathfrak{p}} \bar{\sigma}^{2} \in \mathfrak{O}_{k, \mathfrak{p}}\left[C_{3}\right]^{\times}
$$




\section{N. P. Byott And B. Sodä̈Gui}

to

$$
\tilde{\kappa}_{\mathfrak{p}}=a_{0, \mathfrak{p}}+a_{1, \mathfrak{p}} \sigma+a_{2, \mathfrak{p}} \sigma^{2} \in \mathfrak{O}_{k, \mathfrak{p}}[\Gamma]^{\times} .
$$

Then $\operatorname{Det}\left(\tilde{\kappa}_{\mathfrak{p}}\right)(\chi)=\operatorname{Det}\left(\kappa_{\mathfrak{p}}\right)(\underline{\chi})$ for $\chi=\chi_{0}, \chi_{1}, \chi_{1}^{2}$. Thus for some $w \in \mathbb{J}(k)$ we have

$$
\left(x^{-1} x^{\prime}, w\right)=(\alpha, 1) \operatorname{Det}\left(\left(\tilde{\kappa}_{\mathfrak{p}}\right)_{\mathfrak{p}}\right) \in\left(K^{\times} \times k^{\times}\right) \operatorname{Det}^{0}\left(\mathbb{U}\left(\mathfrak{O}_{k}[\Gamma]\right)\right) \subset \mathbb{J}(K) \times \mathbb{J}(k) .
$$

By (2.3), it follows that $(x, y)\left(x^{-1} x^{\prime}, w\right)$ represents the same class in $\mathrm{Cl}^{0}\left(\mathfrak{O}_{k}[\Gamma]\right)$ as $(x, y)$, so we may take $y^{\prime}=y w$.

Replacing $(x, y)$ by $\left(x^{\prime}, y^{\prime}\right)$, we assume from now on that $x$ is given by (3.4). In particular we have $x_{\mathfrak{p}}=1$ for all $\mathfrak{p}$ above 2 .

Let $\Delta(E / k)$ denote the discriminant ideal of $E / k$. Then $\Delta(E / k)$ is the square of an ideal $\Delta(E / k)^{1 / 2}$ of $\mathfrak{O}_{k}$, since $E / k$ has odd order. As $E / k$ is (totally) ramified at some place of $k$, a slight generalization of [Was96, Theorem 10.1] shows that the norm from $\mathrm{Cl}_{8}\left(\mathfrak{O}_{E}\right)$ to $\mathrm{Cl}_{8}\left(\mathfrak{O}_{k}\right)$ is surjective. Let $\mathfrak{a}$ be a ray class in $\mathrm{Cl}_{8}\left(\mathfrak{O}_{E}\right)$ whose norm in $\mathrm{Cl}_{8}\left(\mathfrak{O}_{k}\right)$ is the class $\left(\Delta(E / k)^{1 / 2}\right)(y)^{-1}$, where $(y)$ denotes the class determined by $y$. Choose prime ideals $\mathfrak{q}_{1}, \mathfrak{q}_{2}$ of $\mathfrak{O}_{E}$, totally split over $k$, and above different ideals in $\mathfrak{O}_{k}$, such that $\mathfrak{q}_{1}$ is in the class $\mathfrak{a}$ of $\mathrm{Cl}_{8}\left(\mathfrak{O}_{E}\right)$, and $\mathfrak{q}_{2}$ is in the same class as $\mathfrak{q}_{1}^{-1-\bar{\sigma}}$ in $\mathrm{Cl}_{64}\left(\mathfrak{O}_{E}\right)$. This is possible by the Tchebotarev density theorem for ray class groups. We then have

$$
\left(N_{E / k}\left(\mathfrak{q}_{1}\right)\right)=\left(\Delta(E / k)^{1 / 2}\right)(y)^{-1} \quad \text { in } \mathrm{Cl}_{8}\left(\mathfrak{O}_{k}\right)
$$

and

$$
\mathfrak{q}_{1}^{1+\bar{\sigma}} \mathfrak{q}_{2}=m \mathfrak{O}_{E},
$$

where $m \equiv 1\left(\bmod ^{*} 64 \mathfrak{O}_{E}\right)$.

Next put $n=m \bar{\sigma}(m)$. Then

$$
n \equiv 1\left(\bmod ^{*} 64 \mathfrak{O}_{E}\right),
$$

and

$$
n \mathfrak{O}_{E}=\left(\mathfrak{q}_{1}^{1+\bar{\sigma}} \mathfrak{q}_{2}\right)^{1+\bar{\sigma}}=\left(\mathfrak{q}_{1}^{\bar{\sigma}}\right)^{2} \mathfrak{q}_{1}^{1+\bar{\sigma}^{2}} \mathfrak{q}_{2}^{1+\bar{\sigma}}
$$

Since $\mathfrak{q}_{1}, \mathfrak{q}_{1}^{\bar{\sigma}^{2}}, \mathfrak{q}_{2}, \mathfrak{q}_{2}^{\bar{\sigma}}$ are distinct prime ideals in $\mathfrak{O}_{E},(3.8)$ shows that $n$ cannot be a square in $E$. On the other hand, $N_{E / k}(n)=N_{E / k}(m)^{2}$ is a square in $k$. It follows from [GS02, Lemme 3.1] that $N=E(\sqrt{n}, \sqrt{\bar{\sigma}(n)})$ is Galois over $k$ with group $A_{4}$. We identify $\operatorname{Gal}(N / k)$ with $\Gamma=\langle\sigma, \tau, \nu\rangle$, where $\sigma$ is an extension of $\bar{\sigma}$ to $N$ and $\tau, \nu \in \operatorname{Gal}(N / E)$ fix $\sqrt{\sigma(n)}$ and $\sqrt{n}$, respectively. By (3.7), the biquadratic extension $N / E$ is tamely ramified and all places of $E$ above 2 split completely in $N$. (Indeed, the corresponding congruence with modulus $8 \mathfrak{O}_{E}$ would be sufficient to guarantee this.)

\section{Resolvents for the biquadratic extension $N / E$}

The group algebra $k[\Delta]$ contains the four primitive idempotents

$$
\begin{aligned}
& e_{0}=\frac{1}{4}(1+\nu+\tau+\nu \tau), \\
& e_{1}=\frac{1}{4}(1+\nu-\tau-\nu \tau), \\
& e_{2}=\frac{1}{4}(1-\nu+\tau-\nu \tau), \\
& e_{3}=\frac{1}{4}(1-\nu-\tau+\nu \tau) .
\end{aligned}
$$

Let

$$
\eta=\frac{1}{4}(1+\sqrt{n})(1+\sqrt{\sigma(n)}) .
$$


Then

$$
e_{0} \eta=\frac{1}{4}, \quad e_{1} \eta=\frac{1}{4} \sqrt{n}, \quad e_{2} \eta=\frac{1}{4} \sqrt{\sigma(n)}, \quad e_{3} \eta=\frac{1}{4} \sqrt{n \sigma(n)} .
$$

Furthermore, $\eta$ is a normal basis for $N / E$, and, since $n \equiv \sigma(n) \equiv 1\left(\bmod ^{*} 4 \mathfrak{O}_{E}\right)$ by $(3.7), \eta$ is also a local normal integral basis at each place above 2 . We will specify corresponding local normal integral bases $\eta_{\mathfrak{p}}$ at each place $\mathfrak{p}$ of $E$.

From (3.8) we have

$$
\begin{aligned}
n \mathfrak{O}_{E} & =\left(\mathfrak{q}_{1}^{\sigma}\right)^{2} \mathfrak{q}_{1}^{1+\sigma^{2}} \mathfrak{q}_{2}^{1+\sigma}, \\
\sigma(n) \mathfrak{O}_{E} & =\left(\mathfrak{q}_{1}^{\sigma^{2}}\right)^{2} \mathfrak{q}_{1}^{\sigma+1} \mathfrak{q}_{2}^{\sigma+\sigma^{2}}, \\
n \sigma(n) \mathfrak{O}_{E} & =\left(\mathfrak{q}_{1}^{1+\sigma+\sigma^{2}} \mathfrak{q}_{2}^{\sigma}\right)^{2} \mathfrak{q}_{1}^{\sigma+\sigma^{2}} \mathfrak{q}_{2}^{1+\sigma^{2}} .
\end{aligned}
$$

For each place $\mathfrak{p}$, fix a uniformizer $\pi(\mathfrak{p})$ of $\mathfrak{O}_{E, \mathfrak{p}}$. Define

$$
\begin{aligned}
\eta_{\mathfrak{q}_{1}} & =\left(e_{0}+e_{1}+e_{2}+\pi\left(\mathfrak{q}_{1}\right)^{-1} e_{3}\right) \eta, \\
\eta_{\mathfrak{q}_{1}^{\sigma}} & =\left(e_{0}+\pi\left(\mathfrak{q}_{1}^{\sigma}\right)^{-1} e_{1}+e_{2}+\pi\left(\mathfrak{q}_{1}^{\sigma}\right)^{-1} e_{3}\right) \eta, \\
\eta_{\mathfrak{q}_{1}^{\sigma}} & =\left(e_{0}+e_{1}+\pi\left(\mathfrak{q}_{1}^{\sigma^{2}}\right)^{-1} e_{2}+\pi\left(\mathfrak{q}_{1}^{\sigma^{2}}\right)^{-1} e_{3}\right) \eta, \\
\eta_{\mathfrak{q}_{2}^{\sigma}} & =\left(e_{0}+e_{1}+e_{2}+\pi\left(\mathfrak{q}_{2}^{\sigma}\right)^{-1} e_{3}\right) \eta, \\
\eta_{\mathfrak{p}} & =\eta \quad \text { for } \mathfrak{p} \neq \mathfrak{q}_{1}, \mathfrak{q}_{1}^{\sigma}, \mathfrak{q}_{1}^{\sigma^{2}}, \mathfrak{q}_{2}^{\sigma} .
\end{aligned}
$$

Then $\mathfrak{O}_{N, \mathfrak{p}}=\mathfrak{O}_{E, \mathfrak{p}}[\Delta] \eta_{\mathfrak{p}}$ for all $\mathfrak{p}$.

Recall that $\phi$ is the irreducible character of $\Delta$ with kernel $\langle\nu\rangle$. The Fröhlich-Lagrange resolvent $\langle\eta, \phi\rangle_{N / E}$ coincides with the usual Lagrange resolvent, and we calculate

$$
\langle\eta, \phi\rangle_{N / E}=4 e_{1} \eta=\sqrt{n} .
$$

Similarly, $\left\langle\eta_{\mathfrak{p}}, \phi\right\rangle_{N / E}=\sqrt{n}$ for all $\mathfrak{p} \neq \mathfrak{q}_{1}^{\sigma}$, and $\left\langle\eta_{\mathfrak{q}_{1}^{\sigma}}, \phi\right\rangle_{N / E}=\pi\left(\mathfrak{q}_{1}^{\sigma}\right)^{-1} \sqrt{n}$. We have therefore established the following lemma.

LEMMA 4.1. With the above notation, the idèle of $E$ given by

$$
\left(\left\langle\eta_{\mathfrak{p}}, \phi\right\rangle_{N / E}\right)_{\mathfrak{p}}\langle\eta, \phi\rangle_{N / E}^{-1}
$$

has content $\left(\mathfrak{q}_{1}^{\sigma}\right)^{-1}$.

\section{Resolvents for $N / k$ and proof of the Theorem}

To complete the proof of the Theorem, we will use Fröhlich's formula for the behaviour of resolvents under induction of characters (see [Frö76, Theorem 7] or [Frö77, Theorem 12]). Recall that $\chi_{2}=$ $\operatorname{Ind}_{\Delta}^{\Gamma} \phi$ and that we have chosen normal bases $\eta$ and $c$ for $N / E$ and $E / k$, respectively. Let $\alpha$ be any normal basis for $N / k$. In our setting, Fröhlich's formula states that

$$
\left\langle\alpha, \chi_{2}\right\rangle_{N / k} \phi(\lambda)=N_{E / k}\left(\langle\eta, \phi\rangle_{N / E}\right) e(E / k),
$$

for some $\lambda \in k[\Delta]^{\times}$, where we may take $e(E / k)^{2} \in k^{\times}$to be the discriminant of the $k$-basis $\left\{\sigma^{i}(c)\right\}_{0 \leqslant i \leqslant 2}$ of $E$. In fact $e(E / k) \in k^{\times}$since the cubic extension $E / k$ is normal. An examination of the proof of Fröhlich's formula shows that $\lambda$ is then the determinant of the matrix $\left(\lambda_{i j}\right)$ over $k[\Delta]$ given by

$$
\sigma^{i}(c) \eta=\sum_{j=0}^{2} \lambda_{i j} \sigma^{j}(\alpha) \text { for } 0 \leqslant i \leqslant 2
$$




\section{N. P. Byott And B. Sodä̈Gui}

For the local normal integral bases $\alpha_{\mathfrak{p}}$ and $\eta_{\mathfrak{p}}$, there is the corresponding formula

$$
\left\langle\alpha_{\mathfrak{p}}, \chi_{2}\right\rangle_{N / k} \phi\left(\lambda_{\mathfrak{p}}\right)=N_{E / k}\left(\left\langle\eta_{\mathfrak{p}}, \phi\right\rangle_{N / E}\right) e_{\mathfrak{p}}(E / k),
$$

with $\lambda_{\mathfrak{p}} \in \mathfrak{O}_{k, 2}[\Delta]^{\times}$and $e_{\mathfrak{p}}(E / k) \in \mathfrak{O}_{k, \mathfrak{p}}^{\times}$obtained analogously. In particular

$$
e_{\mathfrak{p}}(E / k)^{2} \mathfrak{O}_{k, \mathfrak{p}}=\Delta(E / k) \mathfrak{O}_{k, \mathfrak{p}} .
$$

Following the pattern already adopted for $c$ and $\eta$, we will choose $\alpha$ and the $\alpha_{\mathfrak{p}}$ so that $\alpha_{\mathfrak{p}}=\alpha$ for places $\mathfrak{p}$ above 2 . We will further ensure that, at these places, $\alpha_{\mathfrak{p}}$ gives rise to the $\mathfrak{p}$-component in $x \in \mathbb{J}(K)$, and $\lambda_{\mathfrak{p}}$ is $\mathfrak{p}$-adically close to 1 .

First, let $\mathfrak{p}$ be a place of $k$ not above 2. Let $\alpha_{\mathfrak{p}}^{\prime}$ be any local normal integral basis for $N / k$. Then $\operatorname{Tr}_{N_{\mathfrak{p}} / E_{\mathfrak{p}}}\left(\alpha_{\mathfrak{p}}^{\prime}\right)$ is a local normal integral basis for $E / k$. Since the same is true of $c_{\mathfrak{p}}$, we have $c_{\mathfrak{p}}=\kappa_{\mathfrak{p}} \operatorname{Tr}_{N_{\mathfrak{p}} / E_{\mathfrak{p}}}\left(\alpha_{\mathfrak{p}}^{\prime}\right)$ for some $\kappa_{\mathfrak{p}} \in \mathfrak{O}_{k, \mathfrak{p}}\left[C_{3}\right]^{\times}$. Let $\tilde{\kappa}_{\mathfrak{p}} \in \mathfrak{O}_{k, \mathfrak{p}}[\Gamma]^{\times}$be the lift of $\kappa_{\mathfrak{p}}$, obtained as in $(3.5)$, and set $\kappa_{\mathfrak{p}}^{\prime}=\tilde{\kappa}_{\mathfrak{p}} e_{0}+\left(1-e_{0}\right) \in \mathfrak{O}_{k, \mathfrak{p}}[\Gamma]^{\times}$and $\alpha_{\mathfrak{p}}=\kappa_{\mathfrak{p}}^{\prime} \alpha_{\mathfrak{p}}^{\prime}$. Then $\alpha_{\mathfrak{p}}$ is another local normal integral basis for $N / k$, and

$$
\begin{aligned}
\operatorname{Tr}_{N_{\mathfrak{p}} / E_{\mathfrak{p}}}\left(\alpha_{\mathfrak{p}}\right) & =\left(4 e_{0}\right)\left(\tilde{\kappa}_{\mathfrak{p}} e_{0}+\left(1-e_{0}\right)\right) \alpha_{\mathfrak{p}}^{\prime} \\
& =4 e_{0} \tilde{\kappa}_{\mathfrak{p}} \alpha_{\mathfrak{p}}^{\prime} \\
& =\kappa_{\mathfrak{p}} \operatorname{Tr}_{N_{\mathfrak{p}} / E_{\mathfrak{p}}}\left(\alpha_{\mathfrak{p}}^{\prime}\right) \\
& =c_{\mathfrak{p}} .
\end{aligned}
$$

Thus, by (3.2),

$$
\operatorname{Tr}_{N_{\mathfrak{p}} / k_{\mathfrak{p}}}\left(\alpha_{\mathfrak{p}}\right)=\operatorname{Tr}_{E_{\mathfrak{p}} / k_{\mathfrak{p}}}\left(c_{\mathfrak{p}}\right)=1 .
$$

Fröhlich's formula (5.3) holds for this $\alpha_{\mathfrak{p}}$ and some $\lambda_{\mathfrak{p}} \in \mathfrak{O}_{k, \mathfrak{p}}[\Gamma]^{\times}$.

Next we construct a normal basis $\alpha$ for $N / k$, and a new normal basis $\beta$ for $N / E$, with good behaviour above 2. Let $\mathfrak{O}_{E, 2}$ and $\mathfrak{O}_{N, 2}$ be the semilocal completions at 2 of $\mathfrak{O}_{E}$ and $\mathfrak{O}_{N}$, respectively. Since each place above 2 splits completely in $N / E$, we have $N_{2} \cong E_{2}^{4}$ as Galois algebras over $k_{2}$. Explicitly, we may take this isomorphism to be $z \mapsto(z, \tau(z), \nu(z), \tau \nu(z))$. We identify $\mathfrak{O}_{N, 2}$ with $\mathfrak{O}_{E, 2}^{4}$ via this isomorphism. Then the action of $\Gamma$ on $\mathfrak{O}_{N, 2}=\mathfrak{O}_{E, 2}^{4}$ is given by

$$
\begin{gathered}
\tau\left(z_{1}, z_{2}, z_{3}, z_{4}\right)=\left(z_{2}, z_{1}, z_{4}, z_{3}\right), \quad \nu\left(z_{1}, z_{2}, z_{3}, z_{4}\right)=\left(z_{3}, z_{4}, z_{1}, z_{2}\right), \\
\sigma\left(z_{1}, z_{2}, z_{3}, z_{4}\right)=\left(\sigma\left(z_{1}\right), \sigma\left(z_{4}\right), \sigma\left(z_{2}\right), \sigma\left(z_{3}\right)\right) .
\end{gathered}
$$

Let $\beta^{\prime}, \alpha^{\prime} \in N_{2}$ be the elements

$$
\beta^{\prime}=(1,0,0,0), \quad \alpha^{\prime}=c \beta^{\prime}=(c, 0,0,0) .
$$

Then $\alpha^{\prime}$ and $\beta^{\prime}$ are local normal integral bases for $N / k$ and $N / E$ respectively at all places above 2, and $\operatorname{Tr}_{N / E}\left(\beta^{\prime}\right)=1$.

Now choose $\beta \in N$ with

$$
\beta \equiv \beta^{\prime} \quad\left(\bmod 8 \mathfrak{O}_{N, 2}\right), \quad \operatorname{Tr}_{N / E}(\beta)=1,
$$

and set $\alpha=c \beta$. Then

$$
\mathfrak{O}_{k, 2}[\Gamma] \alpha+8 \mathfrak{O}_{N, 2}=\mathfrak{O}_{k, 2}[\Gamma] \alpha^{\prime}+8 \mathfrak{O}_{N, 2}=\mathfrak{O}_{N, 2} .
$$

Thus $\mathfrak{O}_{N, 2}=\mathfrak{O}_{k, 2}[\Gamma] \alpha$ by Nakayama's Lemma, so $\alpha$ is a local normal integral basis for $N / k$ at all places above 2. Similarly, $\beta$ is a local normal integral basis for $N / E$ at these places. Moreover,

$$
\operatorname{Tr}_{N / E}(\alpha)=c \operatorname{Tr}_{N / E}(\beta)=c
$$

and hence, by (3.3),

$$
\operatorname{Tr}_{N / k}(\alpha)=\operatorname{Tr}_{E / k}(c)=1
$$




\section{Realizable Galois module Classes}

Since $\sigma(\beta) \equiv \sigma\left(\beta^{\prime}\right)=\beta^{\prime} \equiv \beta\left(\bmod 8 \mathfrak{O}_{N, 2}\right)$, we also have

$$
\sigma^{j}(c) \beta \equiv \sigma^{j}(c) \sigma^{j}(\beta)=\sigma^{j}(\alpha) \quad\left(\bmod 8 \mathfrak{O}_{N, 2}\right) \quad \text { for } 0 \leqslant j \leqslant 2 .
$$

We take $\alpha$ as our normal basis for $N / k$ in (5.1). For places $\mathfrak{p}$ above 2 , we set

$$
\alpha_{\mathfrak{p}}=\alpha, \quad \lambda_{\mathfrak{p}}=\lambda, \quad e_{\mathfrak{p}}(E / k)=e(E / K),
$$

where $\lambda$ and $e(E / k)$ are as in (5.1). For these places, $\alpha_{\mathfrak{p}}$ is a local normal integral basis and $c_{\mathfrak{p}}=c$. Thus (5.3) now holds for all places $\mathfrak{p}$.

Proposition 5.1. With the above notation, $\lambda \equiv 1\left(\bmod 8 \mathfrak{D}_{k, 2}[\Delta]\right)$.

Proof. As both $\eta$ and $\beta^{\prime}$ are local normal integral bases for $N / E$ above 2 , there is some $\mu^{\prime} \in \mathfrak{O}_{E, 2}[\Delta]^{\times}$ such that

$$
\beta^{\prime}=\mu^{\prime} \eta
$$

We calculate $\mu^{\prime}$ explicitly. By (3.7) there is an element $f \in \mathfrak{O}_{E, 2}$ with $f \equiv 1\left(\bmod 32 \mathfrak{O}_{E, 2}\right)$ and $n=f^{2}$. Under the identification $\mathfrak{O}_{N, 2} \cong \mathfrak{O}_{E, 2}^{4}$ we then have $\sqrt{n}=(f,-f, f,-f)$ and $\sqrt{\sigma(n)}=$ $(\sigma(f), \sigma(f),-\sigma(f),-\sigma(f))$. Thus

$$
\begin{aligned}
& e_{0} \eta=\frac{1}{4}(1,1,1,1), \\
& e_{1} \eta=\frac{1}{4}(f,-f, f,-f), \\
& e_{2} \eta=\frac{1}{4}(\sigma(f), \sigma(f),-\sigma(f),-\sigma(f)), \\
& e_{3} \eta=\frac{1}{4}(f \sigma(f),-f \sigma(f),-f \sigma(f), f \sigma(f)) .
\end{aligned}
$$

Hence $\beta^{\prime}=e_{0} \eta+f^{-1} e_{1} \eta+\sigma(f)^{-1} e_{2} \eta+f^{-1} \sigma(f)^{-1} e_{3} \eta$, so that

$$
\mu^{\prime}=e_{0}+f^{-1} e_{1}+\sigma(f)^{-1} e_{2}+f^{-1} \sigma(f)^{-1} e_{3} \equiv 1 \quad\left(\bmod 8 \mathfrak{O}_{E, 2}[\Delta]\right),
$$

since $f \equiv \sigma(f) \equiv 1\left(\bmod 32 \mathfrak{O}_{E, 2}\right)$.

From $(5.7)$ and $(5.12)$ we now have $\beta \equiv \eta\left(\bmod 8 \mathfrak{O}_{N, 2}\right)$, and hence by $(5.10)$,

$$
\sigma^{j}(c) \eta \equiv \sigma^{j}(\alpha)\left(\bmod 8 \mathfrak{O}_{N, 2}\right) \text { for } 0 \leqslant j \leqslant 2 .
$$

It follows that the matrix $\left(\lambda_{i j}\right)$ defined by (5.2) is congruent modulo $8 \mathfrak{O}_{k, 2}[\Delta]$ to the identity. Hence $\lambda=\operatorname{det}\left(\lambda_{i j}\right) \equiv 1\left(\bmod 8 \mathfrak{O}_{k, 2}[\Delta]\right)$.

Combining the local integral identities (5.3) with the identity (5.1) at the level of fields, we obtain the idèlic equation

$$
\frac{\left(\left\langle\alpha_{\mathfrak{p}}, \chi_{2}\right\rangle_{N / k}\right)_{\mathfrak{p}}}{\left\langle\alpha, \chi_{2}\right\rangle_{N / k}}\left(\frac{\left(\phi\left(\lambda_{\mathfrak{p}}\right)\right)_{\mathfrak{p}}}{\phi(\lambda)}\right)=N_{E / k}\left(\frac{\left(\left\langle\eta_{\mathfrak{p}}, \phi\right\rangle_{N / E}\right)_{\mathfrak{p}}}{\langle\eta, \phi\rangle_{N / E}}\right)\left(\frac{\left(e_{\mathfrak{p}}(E / k)\right)_{\mathfrak{p}}}{e(E / k)}\right)
$$

in $\mathbb{J}(k)$.

Lemma 5.2. The idèle $y^{\prime}=\left(\left\langle\alpha_{\mathfrak{p}}, \chi_{2}\right\rangle_{N / k}\right)_{\mathfrak{p}}\left\langle\alpha, \chi_{2}\right\rangle_{N / k}^{-1} \in \mathbb{J}(k)$ determines the same class in $\mathrm{Cl}_{8}\left(\mathfrak{O}_{k}\right)$ as $y$.

Proof. By Proposition 5.1 and (5.11), we have $\left(\phi\left(\lambda_{\mathfrak{p}}\right)\right)_{\mathfrak{p}} \phi(\lambda)^{-1} \in k^{\times} \mathbb{U}_{8}\left(\mathfrak{O}_{k}\right)$. The content of the idèle $\left(e_{\mathfrak{p}}(E / k)^{2}\right)_{\mathfrak{p}}$ is the discriminant $\Delta(E / k)$ by (5.4). So, using (5.13) and Lemma 4.1, we see that $y^{\prime}$ determines the same class in $\mathrm{Cl}_{8}\left(\mathfrak{O}_{k}\right)$ as $N_{E / k}\left(\left(\mathfrak{q}_{1}^{-1}\right)^{\sigma}\right) \Delta(E / k)^{1 / 2}=N_{E / k}\left(\mathfrak{q}_{1}\right)^{-1} \Delta(E / k)^{1 / 2}$. By (3.6), this is precisely the class determined by $y$. 


\section{Realizable Galois module Classes}

Proof of the Theorem. It suffices to show that $\left(\mathfrak{O}_{N}\right) \in \mathrm{Cl}^{0}\left(\mathfrak{O}_{k}[\Gamma]\right)$ is the class determined by the given pair of idèles $(x, y) \in \mathbb{J}(K) \times \mathbb{J}(k)$. Now $\left\langle\alpha, \chi_{0}\right\rangle_{N / k}=\operatorname{Tr}_{N / k}(\alpha)=1$ and $\left\langle\alpha_{\mathfrak{p}}, \chi_{0}\right\rangle_{N / k}=$ $\operatorname{Tr}_{N_{\mathfrak{p}} / k_{\mathfrak{p}}}\left(\alpha_{\mathfrak{p}}\right)=1$ for all $\mathfrak{p}$ by (5.6) and (5.9). Thus, under the isomorphism (2.3), we can calculate $\left(\mathfrak{O}_{N}\right)$ by taking resolvents of $\alpha$ and the $\alpha_{\mathfrak{p}}$. More precisely, $\left(\mathfrak{O}_{N}\right)$ is represented by $\left(x^{\prime}, y^{\prime}\right)$, where $y^{\prime}$ is as in Lemma 5.2 and $x^{\prime} \in \mathbb{J}(K)$ is obtained by taking resolvents for the character $\chi_{1}$ (if $\zeta \notin k$ ) or the characters $\chi_{1}$ and $\chi_{1}^{2}$ (if $\zeta \in k$ ). Using (5.5) and (5.8), we have

$$
\left\langle\alpha, \chi_{1}^{i}\right\rangle_{N / k}=\left\langle\operatorname{Tr}_{N / E}(\alpha), \underline{\chi}_{1}^{i}\right\rangle_{E / k}=\left\langle c, \underline{\chi}_{1}^{i}\right\rangle_{E / k}
$$

for $i=1,2$, and similarly for the $\left\langle\alpha_{\mathfrak{p}}, \chi_{1}^{i}\right\rangle_{N / k}$. Hence $x^{\prime}$ is as given in (3.4), and we have $x=x^{\prime}$ by the assumption after Proposition 3.1. Thus $\left(\mathfrak{O}_{N}\right)$ is represented by $\left(x, y^{\prime}\right)$. But by Lemma 5.2 and Proposition 2.1, $\left(x, y^{\prime}\right)$ and $(x, y)$ determine the same class in $\mathrm{Cl}^{0}\left(\mathfrak{O}_{k}[\Gamma]\right)$.

\section{REFERENCES}

BS05 N. P. Byott and B. Sodaïgui, Galois module structure for dihedral extensions of degree 8: realizable classes over the group ring, J. Number Theory, to appear.

Frö76 A. Fröhlich, Arithmetic and Galois module structure for tame extensions, J. reine angew. Math. 286/287 (1976), 380-440.

Frö77 A. Fröhlich, Galois module structure, in Algebraic number fields (L-functions and Galois properties), ed. A. Fröhlich (Academic Press, London, 1977), 133-191.

Frö83 A. Fröhlich, Galois module structure of algebraic integers (Springer, Berlin, 1983).

GS02 M. Godin and B. Sodaïgui, Classes de Steinitz d'extensions à groupe de Galois $A_{4}$, J. Théor. Nombres Bordeaux 14 (2002), 241-248.

GS03 M. Godin and B. Sodaïgui, Realizable classes of tetrahedral extensions, J. Number Theory $\mathbf{9 8}$ (2003), 320-328.

McC83 L. R. McCulloh, Galois module structure of elementary abelian extensions, J. Algebra 82 (1983), $102-134$.

McC87 L. R. McCulloh, Galois module structure of abelian extensions, J. reine angew. Math. 375/376 (1987), 259-306.

Sod97 B. Sodaïgui, Classes réalisables par des extensions métacycliques non abéliennes et éléments de Stickelberger, J. Number Theory 65 (1997), 87-95.

Sod99 B. Sodaïgui, 'Galois module structure' des extensions quaternioniennes de degré 8, J. Algebra 213 (1999), 549-556.

Sod00 B. Sodaïgui, Relative Galois module structure and Steinitz classes of dihedral extensions of degree 8, J. Algebra 223 (2000), 367-378.

Sov99 E. Soverchia, Galois module structure in metacyclic extensions, Preprint (1999).

Was96 L. C. Washington, Introduction to cyclotomic fields, Graduate Texts in Mathematics, vol. 83, second edition (Springer, New York, 1996).

Nigel P. Byott n.p.byott@ex.ac.uk

Department of Mathematical Sciences, University of Exeter, Exeter EX4 4QE, UK

Bouchaïb Sodaïgui bouchaib.sodaigui@univ-valenciennes.fr

Département de Mathématiques, Université de Valenciennes, Le Mont Houy, 59313 Valenciennes cedex 9, France 